\title{
Anaesthetic Management of a Patient with a Pituitary Tumour and Combined Deficiency of Factor VII and IX: Controversies of a Clinical Case \\ Oliveira, $M^{*}$; Caria, $T^{*}$; Neto, $M^{* *}$; Reis, $A^{* *}$; Palma Mira, $F^{* *}$; André, $A^{* *}$ \\ ${ }^{*}$ Resident; ${ }^{* *}$ Attending Anaesthesiologist \\ Centro Hospitalar Lisboa Ocidental, Anaesthesiology Department \\ Lisbon, Portugal.
}

\section{Background}

Inherited bleeding and clotting disorders are rare but sometimes encountered by anaesthetists during emergency or elective procedures. The perioperative management of these uncommon conditions can be challenging. ${ }^{(1)}$

\section{Case Report}

We report the case of a 28 -year-old-man, scheduled for the excision of a pituitary tumour by endonasal approach. His medical history included pan hypopituitarism, $\beta$-thalassemia, and mild deficit of factor VII and IX. He has had two surgical interventions for the excision of the tumour and both had to be interrupted due to difficulties in the surgical approach owing to bleeding.

This time, the case was managed in collaboration with the imunohemotherapy team. Investigation of the patient coagulation status showed factor VII activity of 33\% and factor IX activity of $61 \%$.

Despite his low factor VII deficiency assay, he was given recombinant factor VIIa (NovoSeven ${ }^{\circledR}$ ) 1g, i.v. $30 \mathrm{~min}$ before surgery. During the procedure, due to continuous bleeding, two doses of factor VIIa were given (at 4 hour intervals) and a low-dose aminocaproic acid perfusion was started.

Notwithstanding all this measures, due too poor visualization, the surgery was converted in open craniotomy.

The coagulation control revealed an important procoagulant state.

By the end of the procedure the surgeon noticed bilateral fixed mydriasis and either a thrombotic event or surgical complication was hypothesized. CT- scan revealed no alterations. The patient was transferred to the intensive care unit under mechanical ventilation. The patient fully recovered and was discharged without any neurological deficits.

\section{Discussion}

Management of patients with factor VII deficiency consists of factor replacement therapy either prophylactically or to treat acute bleeding episode.

Levels of more than $10 \%$ are usually considered haemostatic although it is difficult to predict the perioperative risk of bleeding due to a poor correlation between FVII activity and severity of bleeding. ${ }^{(1)(2)}$

Although it is not indicated to correct a mild deficit, in our case, due to the previous bleeding history, we decided, in collaboration with the imunohemotherapy team, to give recombinant factor VIIa prophylactically to improve the surgical conditions. Despite this measure, during the procedure, it was necessary to give two more doses of recombinant factor VIIa and aminocaproic acid, which may have triggered a procoagulant state.

Treatment with haemostatics and antifibrinolytic agents carries the risk of thrombotic events and close motorization is fundamental.

\section{Learning Points}

Providing good surgical conditions while maintaining patient safety can be challenging. In cases in which haemostatics and antifibrinolytic agents are used close monitoring of coagulation status is essential.
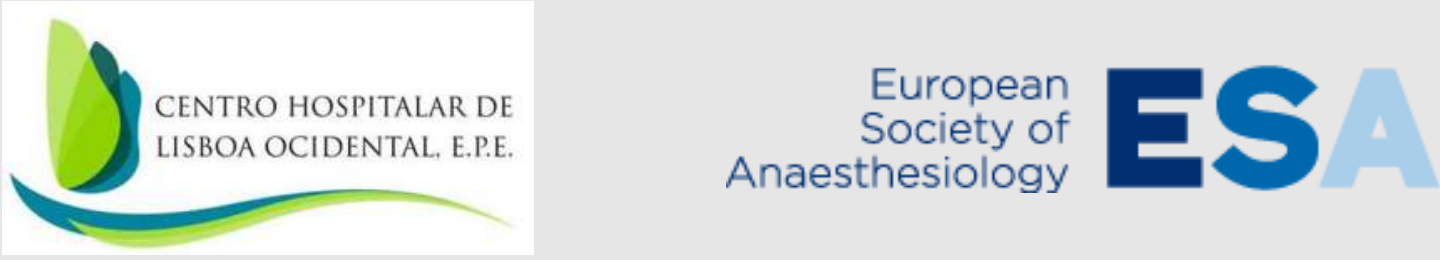

1- R. Mandhyan, A; Tiwari and G. Cherian; Congenital factor VII deficiency; BJA 2010; Volume 104, Issue 2; Pp. 267-268

2- Yoshida A et all; Anesthetic management of a patient with factor VII deficiency undergoing laparoscopic colectomy: a case report; JA Clinical Reports 2016 